\title{
La lesión miocárdica en el perioperatorio de cirugía no cardíaca
}

\section{Myocardial injury in the perioperative period of non-cardiac surgery}

Dr. Pastor Luna-Ortiz, * Dra. Nora Bernal-Ríos, ${ }^{\ddagger}$ Dra. Mariela García-Bravo, Dr. Luis Leobardo Fortis-Olmedo, ${ }^{\ddagger}$ Dr. Martin Martínez-Rosas ${ }^{\S}$

Citar como: Luna-Ortiz P, Bernal-Ríos N, García-Bravo M, Fortis-Olmedo LL, Martínez-Rosas M. La lesión miocárdica en el perioperatorio de cirugía no cardíaca. Rev Mex Anestesiol. 2021; 44 (2): 123-129. https://dx.doi.org/10.35366/99016

RESUMEN. Estudios recientes sobre las causas de muerte en el postoperatorio de cirugía no cardíaca han identificado a la lesión miocárdica como una complicación que se asocia con eventos cardíacos adversos mayores que aumentan la mortalidad a 30 días. La lesión miocárdica se manifiesta como una elevación de las troponinas cardíacas que se produce durante o a los 30 días después de la cirugía, sin que los pacientes presenten síntomas y sin cambios en el electrocardiograma de superficie. En la actualidad, se busca mejorar el diagnóstico oportuno de esta complicación y desarrollar terapias preventivas. En esta revisión abordamos la evidencia de esta lesión, sus mecanismos fisiopatológicos y su manejo.

ABSTRACT. Recent studies on the death causes in the postoperative period of non-cardiac surgery have identified myocardial injury as a complication that is associated with major adverse cardiac events that increase mortality at 30 days after surgery. This kind of myocardial lesion is characterized by the elevation of the cardiac troponins levels during or in the 30 days after the surgery, without symptoms ischemia or changes in the electrocardiogram. Currently, one main goal has been the timely diagnosis of this complication, besides preventive therapies development. The present review article examines the current body of knowledge of this injury, the physiopathological mechanisms and its management.

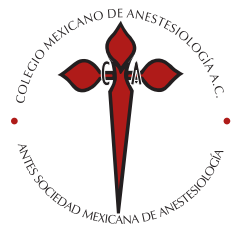

Palabras clave:

Lesión miocárdica, cirugía no cardíaca, elevación de troponinas, isquemia miocárdica.

Keywords:

Myocardial injury, non-cardiac surgery, troponin high levels, myocardial ischemia.

* Departamento de Farmacología. Instituto Nacional de Cardiología «Ignacio Chávez». Facultado BLS y ACLS por AHA; Instructor BLS y ACLS por AHA.

‡ Departamento de Anestesiología. American British Cowdray Hospital. $\S$ Departamento de Fisiología. Instituto Nacional de Cardiología «Ignacio Chávez».

Correspondencia:

Dr. Pastor Luna-Ortiz Instituto Nacional de Cardiología «Ignacio Chávez», Departamento de Farmacología. Juan Badiano 1, Belisario Domínguez Secc. 16, Tlalpan, 14080, CDMX. E-mail:pastor.luna05@gmail.com

Recibido: 19-07-2019

Aceptado:15-07-2020

\section{INTRODUCCIÓN}

$\mathrm{E}$ studios recientes sobre las causas de muerte en el -1 perioperatorio de cirugía no cardíaca (CNC) han identificado un nuevo concepto clínico, el término: lesión miocárdica en cirugía no cardíaca (MINS por sus siglas en inglés). La MINS se define como la lesión miocárdica, manifestada por una elevación de las troponinas cardíacas (Tnc) que se produce durante o a los 30 días después de una CNC sin que los pacientes presenten síntomas y sin cambios en el electrocardiograma de superficie $(E C G)^{(1)}$. Existe estrecha asociación entre los niveles de las Tnc y la mortalidad a corto y largo plazo ${ }^{(2,3)}$. En la actualidad, se busca mejorar el diagnóstico oportuno de la MINS y desarrollar terapias potenciales que la prevengan durante el manejo perioperatorio del cardiópata en CNC. En esta revisión abordamos la evidencia de la MINS, sus mecanismos fisiopatológicos y el manejo perioperatorio.

\section{COMPLICACIONES POSTOPERATORIAS EN CIRUGÍA NO CARDÍACA}

Cada año, el $4 \%$ de la población mundial se somete a un procedimiento quirúrgico, de los cuales, el 30\% tiene una cirugía mayor con por lo menos un factor de riesgo cardiovascular ${ }^{(4)}$. La mortalidad a 30 días de estos pacientes es de $0.5-2.0 \%{ }^{(5)}$. La principal causa de muerte son los eventos cardíacos y cerebrales adversos mayores, y de éstos, la mayoría está representada por la isquemia miocárdica y el infarto al miocardio (IM) ${ }^{(5)}$. El espectro del daño miocárdico, que incluye la isquemia, la 
lesión y el infarto, es más amplio de lo que se pensaba por la aparición de la MINS y la elevación asintomática de las Tnc. La MINS ha sido identificada como una complicación perioperatoria cuya frecuencia no ha sido evaluada aún de manera precisa, pero está asociada fuertemente con la mortalidad a 30 días $^{(6,7)}$. En contraste con el IM perioperatorio tipo 1 por ruptura de la placa aterosclerótica, la MINS frecuentemente no presenta síntomas típicos de isquemia miocárdica, como dolor torácico, angina de pecho o disnea y por lo tanto no se diagnostica en la práctica clínica diaria ${ }^{(6,7)}$.

Considerando que aproximadamente se efectúan 350 millones de cirugías anualmente en el mundo y que más de 75 millones de pacientes experimentarán complicaciones postoperatorias con dos millones de fallecimientos, y que además existe un mayor número de pacientes con riesgo cardiovascular elevado, las estrategias para detectar, tratar y mejorar los resultados deben tener beneficios en los pacientes que presentan MINS. En particular, la detección rápida y confiable de la MINS es el primer paso para lograr mejores resultados de esta complicación perioperatoria en la $\mathrm{CNC}$.

\section{LAS TROPONINAS CARDÍACAS COMO BIOMARCADORES DE LESIÓN MIOCÁRDICA}

Considerando la dificultad del diagnóstico clínico de la MINS por la poca sensibilidad del ECG para su detección, y el mal pronóstico de la lesión miocárdica perioperatoria, se recomienda la determinación de las Tnc en el perioperatorio (antes de la cirugía y 48-72 horas después de ésta) en los pacientes de alto riesgo cardiovascular ${ }^{(6)}$. Con esta estrategia se ha encontrado que la MINS ocurre en el $5-25 \%$ de los pacientes que se someten a CNC. Esta determinación permite no sólo detectar la aparición de la MINS, sino cuantificar el grado de lesión aguda del miocardio en ausencia de signos o síntomas de isquemia en pacientes con elevado riesgo de complicaciones cardiovasculares.

Las troponinas son un complejo de tres proteínas reguladoras contráctiles (troponinas $\mathrm{T}$, I y C) que residen a intervalos regulares en el filamento delgado del músculo estriado que inhibe la contracción al bloquear la interacción de la actina y la miosina. Las troponinas I (TncI) y T (TncT) cardíacas son marcadores muy sensibles y específicos para detectar el daño miocárdico, estos biomarcadores no se encuentran normalmente en la circulación sanguínea. El músculo cardíaco lesionado libera troponina al torrente sanguíneo, por lo que un aumento en el nivel de este biomarcador en plasma está en relación directa con la lesión miocárdica ${ }^{(8)}$. Las troponinas son diferentes entre el músculo esquelético y el cardíaco, lo que permite su uso como un biomarcador cardíaco específico. Muchos pacientes presentan Tnc elevadas después de una $\mathrm{CNC}$, pero sólo el $40 \%$ cumple con la definición universal actual de IM tipo 1 debido a un síndrome coronario agudo $(\mathrm{SCA})^{(9)}$ una parte del $60 \%$ restante podría entrar en la clasificación del MINS; sin embargo, aún no se ha determinado su predominancia debido a la falta de conocimiento del fenómeno y al desarrollo de mejores estrategias de evaluación.

La mayoría de los casos en los que se encuentran Tnc elevadas se han clasificado como IM tipo 2, que se refieren como «isquemia de demanda» producida por un desequilibrio entre la oferta y la demanda de oxígeno miocárdico debido a una estenosis de arteria coronaria fija y a un aumento de la demanda por factores perioperatorios $(\text { Figura } 1)^{(10)}$.

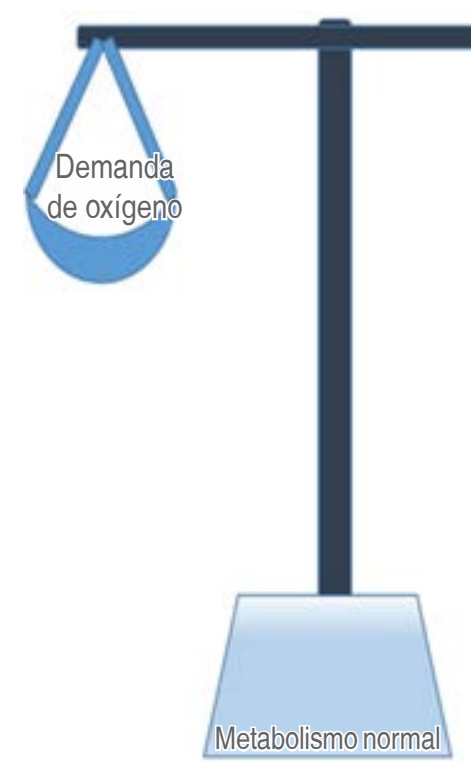

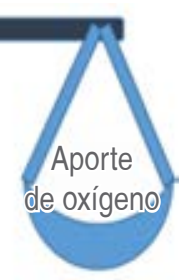
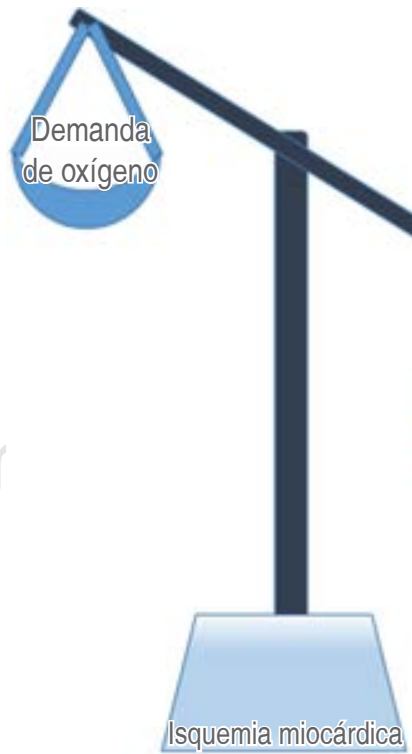

Figura 1:

La isquemia miocárdica ocurre por desbalance entre el aporte y la demanda de oxígeno. 


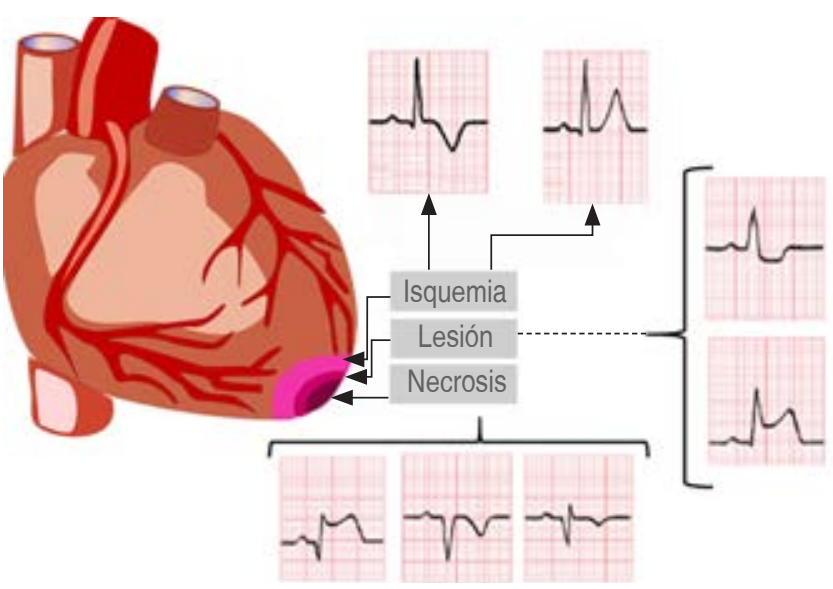

Figura 2: Cambios electrocardiográficos en la isquemia, lesión y necrosis del miocardio.

\section{LA CARDIOPATÍA ISQUÉMICA EN EL PERIOPERATORIO}

La cardiopatía isquémica es una de las principales causas de morbimortalidad en el perioperatorio de la CNC, con una incidencia de 2 a $6 \%{ }^{(11)}$. Se sabe que más de la mitad de los fallecimientos en el postoperatorio son causados por eventos cardíacos y la mayoría son de origen isquémico. La prevención de la isquemia miocárdica perioperatoria es de gran importancia para mejorar los resultados en el postoperatorio ${ }^{(12)}$. En los pacientes con cardiopatía isquémica o con factores de riesgo, la frecuencia de isquemia miocárdica en el perioperatorio reportada es de $20-63 \%{ }^{(12)}$. Varios estudios han demostrado que esta complicación ocurre más frecuentemente en el postoperatorio que en el pre- o intraoperatorio, con una relación aproximada de $3: 1$ y 5:1, respectivamente. La mortalidad a 30 días asociada a CNC con riesgo moderado a alto es entre 2 a $5 \%$ y aumenta a medida que la población envejece y en pacientes con más factores de riesgo y comorbilidades que son sometidos a CNC de alto riesgo.

\section{FISIOPATOLOGÍA DE LA ISQUEMIA MIOCÁRDICA}

La isquemia ocurre cuando la demanda de oxígeno del músculo cardíaco supera al aporte (Figura 1). La cardiopatía isquémica se define como una disminución del flujo sanguíneo coronario que es insuficiente para satisfacer las demandas metabólicas del miocardio. Las condiciones más frecuentes que predisponen a la isquemia miocárdica son la enfermedad de las arterias coronarias, la hipertrofia del ventrículo izquierdo, la estenosis valvular aórtica grave y la hipertensión arterial sistémica. Durante la isquemia miocárdica se produce también una disminución en la eliminación de productos del metabolismo anaeróbico, lo que produce alteraciones de las funciones eléctricas y mecánicas del corazón.
Los cambios en la función eléctrica se pueden detectar en el ECG en el que aparecen cambios de la repolarización del corazón del tipo de la irritabilidad ventricular y de alteraciones en la formación y en la conducción del impulso. Los cambios iniciales de repolarización que se ven en la isquemia miocárdica son cambios en la onda $\mathrm{T}$, si la isquemia se prolonga, se produce depresión o elevación del segmento ST indicativo de lesión miocárdica y la aparición de onda Q indica necrosis del miocardio (Figura 2).

\section{Oferta y demanda de oxígeno miocárdico}

Cuando existe una obstrucción de las arterias coronarias, un aumento en el consumo de oxígeno como ocurre en el ejercicio, en la taquicardia, cuando existe un aumento en la contractilidad o un aumento en la presión arterial, puede producirse un desbalance entre la oferta y la demanda de oxígeno (Figura 3), esta condición se llama «isquemia de demanda» y es responsable de la mayoría de los episodios de angina estable crónica. En otras situaciones, el desbalance es producido por un aumento en el tono vascular coronario o por agregación plaquetaria y formación de trombo; esta condición se llama «isquemia de oferta» y es responsable de la angina inestable y del IM (Figura 3). Los determinantes de la oferta son: el flujo sanguíneo coronario, la presión de perfusión coronaria, la saturación de oxígeno y el contenido de hemoglobina.

\section{Flujo sanguíneo coronario}

El peso promedio del corazón humano es de $350 \mathrm{~g}$, o el $0.5 \%$ del peso corporal total. El flujo sanguíneo coronario normal es de $80 \mathrm{~mL} / 100 \mathrm{~g}$ de tejido cardíaco por minuto en reposo, o el $6 \%$ del gasto cardíaco total; su flujo puede aumentarse varias veces durante el ejercicio. El corazón normalmente obtiene su energía exclusivamente del metabolismo oxidativo aerobio, con un consumo de oxígeno a razón de $10 \mathrm{~mL}$ por $100 \mathrm{~g} / \mathrm{min}$ de tejido miocárdico, esto significa que tiene que extraer $14 \mathrm{~mL}$ de oxígeno de cada $100 \mathrm{~mL}$ de sangre arterial coronaria. El flujo sanguíneo coronario depende del consumo de oxígeno miocárdico. El consumo de oxígeno en reposo es de aproximadamente $200 \mathrm{~mL} / \mathrm{min}$ y durante el ejercicio máximo se puede incrementar a 1,000 $\mathrm{mL} / \mathrm{min}$. El corazón es capaz de controlar su propio flujo sanguíneo según sus necesidades metabólicas, este control es independiente de la presión arterial de perfusión entre los límites de 60 a 180 $\mathrm{mmHg}$. A esto se le llama autorregulación, definida como el mantenimiento del flujo coronario constante para un amplio abanico de presiones de perfusión ${ }^{(13)}$.

La resistencia vascular coronaria está determinada por fuerzas compresivas externas, así como por factores metabólicos, endoteliales y neurales que regulan el tono coronario intrínseco a través de la autorregulación. 
En condiciones de normalidad, el flujo coronario es determinado por el gradiente entre la presión diastólica aórtica y la presión de la aurícula derecha a la que se oponen fundamentalmente resistencias compresivas intramiocárdicas (en especial subendocárdicas) y resistencias del árbol coronario determinadas por el tono vascular de las arteriolas de autorregulación. El lecho coronario puede dilatarse entre cuatro y seis veces en respuesta a las mayores demandas de oxígeno aumentando el flujo coronario, el cual es llamado «reserva de flujo coronario» ${ }^{(14)}$. La extracción basal de oxígeno por el miocardio es muy alta, por lo que el corazón debe recurrir a un aumento del flujo coronario como principal mecanismo de respuesta ante el aumento del trabajo cardíaco. Es de destacar que la resistencia al flujo coronario depende principalmente de las pequeñas arteriolas provistas de músculo liso (50-60\% de la resistencia) y en menor medida de los capilares coronarios (20\% de la resistencia). De esta manera, los vasos epicárdicos juegan un papel menor, funcionando como vasos de conductancia y aportando aproximadamente un $5 \%$ de la resistencia al flujo coronario ${ }^{(14)}$.

\section{Presión de perfusión coronaria}

La presión de perfusión coronaria (PPC) es la diferencia entre la presión diastólica aórtica (PDAo) (ya que el flujo coronario tiene lugar en la diástole) y la presión telediastólica ventricular izquierda (PDFVI) (PPC = PDAo-PDFVI). El valor de PPC normal es de $60 \mathrm{mmHg}$; valores inferiores producen acidosis intramiocárdica, extrasístoles y actividad eléctrica sin pulso ${ }^{(15-17)}$.

La PPC suele ser más elevada que la presión en el seno coronario; el hecho de que el componente distal de la PPC sea más elevado que la presión en el territorio venoso correspondiente es una particularidad del miocardio con respecto a otros órganos. La presión efectiva distal se incrementa del subepicardio al subendocardio cuando aumentan el tono vasomotor y la PDFVI ${ }^{(16,17)}$.

\section{Determinantes de la demanda de oxígeno miocárdica}

La demanda de oxígeno del miocardio aumenta cuando el corazón desarrolla mayor trabajo. Es decir, cuando aumentan: la frecuencia cardíaca, la contractilidad y la tensión de la pared del ventrículo izquierdo (Ley de Laplace). Como ya se mencionó, la perfusión coronaria se produce durante la diástole, por lo que un aumento en la frecuencia cardíaca reduce el tiempo de llenado diastólico. Las taquicardias elevan el consumo de oxígeno y disminuyen la oferta. La contractilidad miocárdica y el estrés de la pared son difíciles de medir, pero se ha demostrado que el consumo de oxígeno por minuto en el corazón es proporcional a un índice de tensión-tiempo por minuto, que corresponde a la presión sistólica media multiplicada por la duración de la sístole y por la frecuencia cardíaca. Por este motivo, en la práctica clínica diaria se utiliza el llamado doble producto (DP), que resulta de la multiplicación de la presión arterial sistólica y la frecuencia cardíaca, $(\mathrm{DP}=\mathrm{PASxFC})$. Tomando en cuenta las frecuencias normales de 60 a 90 latidos por minuto y cifras sistólicas de tensión arterial entre 110 y $130 \mathrm{mmHg}$, los márgenes del doble producto serán normales cuando se encuentren entre 6,600 y 11,700 en reposo. Por arriba de 12,000 es indicativo de isquemia miocárdica ${ }^{(18)}$.

\section{EVENTOS ADVERSOS CARDÍACOS Y CEREBRALES MAYORES (MACCE)}

Los principales eventos adversos cardiovasculares y cerebrovasculares mayores (MACCE, por sus siglas en inglés)

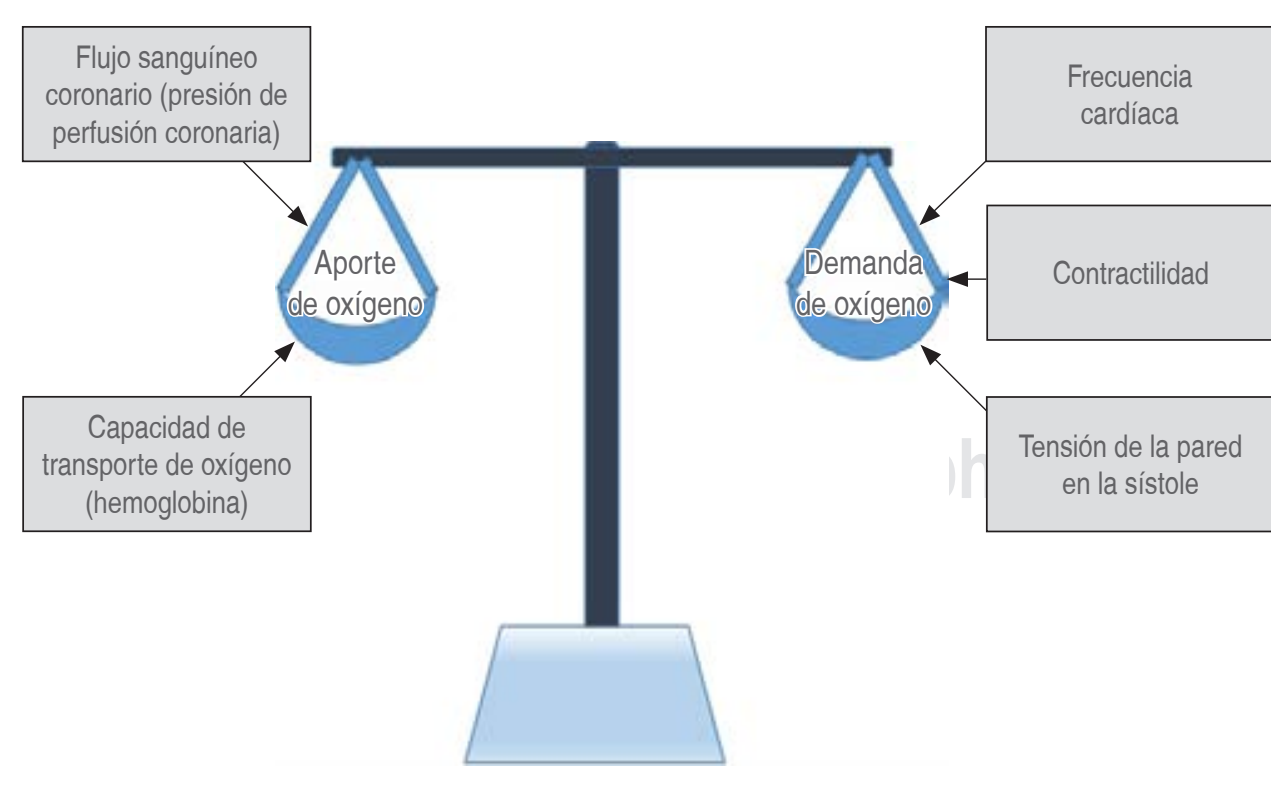

Figura 3:

Factores que determinan el balance entre la oferta y la demanda de oxígeno del miocardio que pueden llevar a isquemia, lesión y/o necrosis. 
incluyen a la muerte cardíaca, la muerte cerebrovascular, el paro cardíaco no fatal, el infarto agudo al miocardio, insuficiencia cardíaca congestiva, arritmias cardíacas, angina y accidente cerebral vascular. Los MACCE constituyen una fuente importante de morbilidad y mortalidad perioperatoria. En un estudio realizado en 3,387 pacientes reclutados de $\mathrm{CNC}$, 146 (4.3\%) desarrollaron al menos un MACCE ${ }^{(19)}$. Los autores identificaron siete factores de riesgo independientes para que ocurran estos eventos: historia de cardiopatía isquémica, historia de insuficiencia cardíaca, enfermedad renal crónica, historia de accidente cerebral vascular, ECG anormal, hipotensión perioperatoria y transfusión sanguínea ${ }^{(19)}$.

Las complicaciones cardiovasculares prolongan la hospitalización, el aumento de los costos médicos y son la principal causa de muerte perioperatoria ${ }^{(20)}$. En las últimas décadas, ha habido mejoras en la estratificación del riesgo perioperatorio además de avances en la técnica quirúrgica y anestésica. Al mismo tiempo, el aumento de la carga de factores de riesgo cardiovascular en la población que se somete a $\mathrm{CNC}$ puede disminuir los avances en los resultados perioperatorios a largo plazo.

En un estudio basado en un Registro Nacional en Estados Unidos entre 2004 a 2013 se encontró que entre 10,581,621 hospitalizaciones por cirugía mayor no cardíaca, el MACCE perioperatorio ocurrió en 317,479 (3.0\%), lo que corresponde a una incidencia anual de aproximadamente $150,000^{(21)}$. Los MACCE ocurrieron con mayor frecuencia en pacientes sometidos a cirugía vascular (7.7\%), torácica (6.5\%), trasplante de órganos $(6.3 \%)$ y la más baja se observó en pacientes sometidos a cirugía ginecológica y obstétrica ${ }^{(21)}$.

Entre 2004 y 2013, la frecuencia de MACCE disminuyó de 3.1 a $2.6 \%$ impulsada por una disminución en la frecuencia de muerte perioperatoria e IM pero con un aumento en el accidente cerebrovascular isquémico perioperatorio de $0.52 \%$ en 2004 a $0.77 \%$ en $2013^{(21)}$. El MACCE perioperatorio fue más frecuente en los hombres 3.5 vs 2.6\% en las mujeres, con mayor mortalidad hospitalaria. El MACCE ocurrió en una de cada 33 hospitalizaciones por $\mathrm{CNC}^{(21)}$. A pesar de las reducciones en la tasa de muerte y el IM entre los pacientes que se someten a cirugía mayor no cardíaca en los Estados Unidos, el ictus isquémico perioperatorio ha aumentado a través del tiempo.

\section{PRONÓSTICO DE LA LESIÓN MIOCÁRDICA EN CIRUGÍA NO CARDÍACA}

Varios estudios resumidos en dos metaanálisis estudiaron la asociación entre las Tnc elevadas en el postoperatorio y la mortalidad. Levy y colaboradores ${ }^{(22)}$ examinaron 14 estudios y 3,139 pacientes. Los niveles de Tnc se relacionaron con la mortalidad a un año y después de un año de la cirugía. Redfern y su equipo ${ }^{(23)}$ estudiaron 1,873 pacientes vasculares

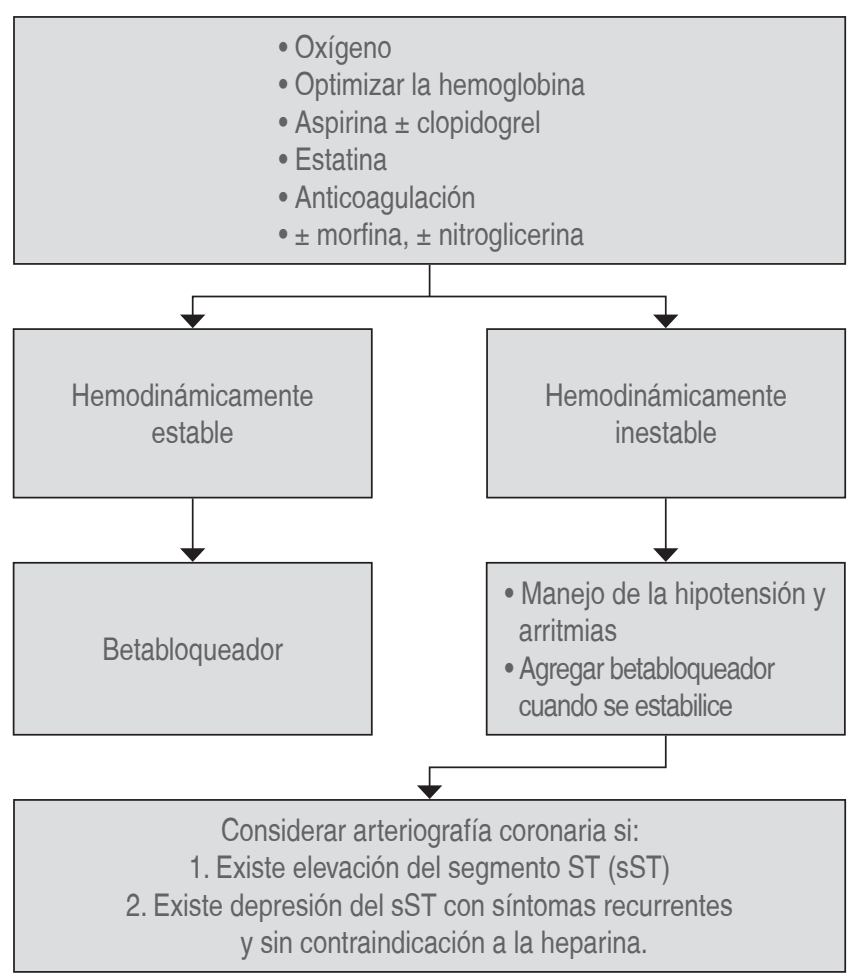

Figura 4: Algoritmo propuesto para el manejo de pacientes con lesión miocárdica después de cirugía no cardíaca. Modificado de: Biccard BM..$^{30}$

en ocho cohortes y encontraron elevación de las Tnc aisladas sin IM asociado a la mortalidad a 30 días. El concepto de MINS nació de un estudio muy importante llamado VISION (Vascular Events in Noncardiac Surgery Patients Evaluation Study) realizado en $2012^{(6)}$. En este estudio se determinaron las concentraciones de troponina $\mathrm{T}$ (TnT) elevadas en el postoperatorio y se valoraron las complicaciones cardiovasculares en pacientes mayores de 45 años intervenidos de CNC, en una cohorte de 15,065 pacientes. Las mediciones de TnT se realizaron a las seis y 12 horas de la cirugía y en los primeros tres días del postoperatorio. Encontrándose que aún en concentraciones bajas, los niveles de TnT son predictivos de mortalidad a los 30 días. En el estudio se incluyeron pacientes intervenidos de manera electiva o de urgencia, con anestesia general o regional. Se observó que los picos de TnT $>0.02 \mathrm{ng} / \mathrm{mL}$ eran predictores independientes de mortalidad de cualquier causa a los 30 días.

El mecanismo de MINS sigue sin estar completamente claro, aunque es probable que sea similar al infarto del miocardio en el perioperatorio. Desde $2007^{(24)}$, así como en la Cuarta Definición Universal del Infarto del Miocardio ${ }^{(25)}$, diferentes tipos de IM se han identificado. Los tipos 1 y 2 son los más importantes en el período perioperatorio de la CNC. El tipo 1 es el clásico IM con ruptura de la placa de ateroma coronario 
y subsecuente trombosis que es la forma más dominante en la población no quirúrgica. La medición de las Tnc no es de gran ayuda para diferenciar entre el IM tipo 1 y tipo $2^{(6,26)}$.

Como ya se mencionó, los pacientes pueden presentar lesión miocárdica en el período perioperatorio que no cumple los criterios diagnósticos de IM. Sin embargo, estos eventos podrían ser indicadores de un mal pronóstico, que la intervención oportuna y apropiada podría potencialmente prevenir.

\section{PREVENCIÓN DE LESIÓN MIOCÁRDICA EN CIRUGÍA NO CARDÍACA}

El primer paso en las medidas de prevención del MINS es la identificación del riesgo y su clasificación ${ }^{(6)}$. Los factores probables que facilitan la isquemia miocárdica tipo 1 son el estrés emocional y fisiológico, la respuesta procoagulante a la cirugía ${ }^{(27)}$, así como los episodios de taquicardia e hipertensión en el perioperatorio, los cuales aumentan las fuerzas de rozamiento con el riesgo asociado de ruptura de la placa aterosclerótica $^{(28,29)}$. El IM tipo 2 es secundario a un desbalance entre la oferta y la demanda, y en la actualidad se sabe que es más aparente en el perioperatorio ${ }^{(30,31)}$, especialmente con el advenimiento de las Tnc más sensibles como biomarcadores.

En las guías de valoración del riesgo cardíaco se recomienda el uso de dos índices: el índice de riesgo cardíaco revisado (IRCR) ${ }^{(32)}$ y el National Surgical Quality Improvement Program (NSQIP) también conocido como el índice de Gupta ${ }^{(33)}$, ambos han sido avalados por la American College of Cardiology (ACC) y por la American Heart Association (AHA). El IRCR fue introducido en 1999 y es el más usado por los anestesiólogos pero el NSQIP introducido en 2011 está basado en una muestra mucho más grande ${ }^{(32)}$.

\section{MANEJO DE LAS MACCE}

Las Guías de la Sociedad Cardiovascular Canadiense (CCS) sobre la valoración del riesgo cardíaco y manejo de pacientes que son sometidos a CNC, guías actualizadas publicadas en $2017^{(34)}$, sugieren primero identificar el riesgo midiendo los niveles de Tnc y después iniciar la terapia intensiva temprana para la lesión miocárdica. Ellos recomiendan medir el péptido natriurético cerebral (PNB), antes de la cirugía para mejorar la valoración del riesgo cardíaco perioperatorio en pacientes mayores de 65 años de edad y en pacientes entre 45 y 64 años de edad con enfermedad cardiovascular importante o en pacientes con IRCR elevado ${ }^{(34)}$.

Las recomendaciones actuales indican suspender la aspirina tres días antes de la cirugía para prevenir el sangrado, a menos que el paciente tenga colocación reciente de stent coronario o cirugía de carótida; suspender los IECA 24 horas antes de la cirugía, iniciar estatinas diarias de tres a siete días antes de la cirugía y continuar los bloqueadores beta si ya los estaban recibiendo antes de la cirugía por cualquier indicación. Sin embargo, se ha establecido que iniciar bloqueadores beta en el preoperatorio reduce los eventos cardíacos adversos, pero aumenta los eventos cerebrovasculares y la mortalidad. Las guías de CCS recomiendan medir las Tnc diariamente por 48 a 72 horas después de la cirugía en pacientes de alto riesgo, pacientes mayores de 45 años con comorbilidades o mayores de 65 años de edad ${ }^{(34)}$.

Las complicaciones son comunes en la primera semana después del MINS, por lo que se requiere una intervención temprana. Los resultados del estudio sobre el tratamiento de lesiones miocárdicas después de la cirugía no cardíaca (MANAGE) $)^{(35)}$ recomiendan usar mejores criterios de valoración primarios. El estudio MANAGE evaluó el impacto de la anticoagulación con dabigatrán en las complicaciones vasculares perioperatorias después de MINS. El dabigatrán $110 \mathrm{mg} / 12$ horas disminuyó el riesgo de complicaciones vasculares mayores postoperatorias sin ningún riesgo de sangrado ${ }^{(35)}$.

La literatura respalda el uso de la administración postoperatoria de aspirina y el tratamiento con estatinas para disminuir la mortalidad a 30 días después del MINS ${ }^{(36)}$. Además, la intensificación de la terapia (definida como la introducción o el aumento de cualquiera de los siguientes grupos de fármacos cardiovasculares: agentes antiplaquetarios, bloqueadores beta, estatinas o IECA) durante el período postoperatorio ha demostrado estar asociada con una mejoría de la sobrevida a un año. Sin embargo, el decidir sobre una terapia específica debe ser parte de un enfoque multidisciplinario. Los beneficios potenciales de las terapias deben valorarse frente a los riesgos potenciales de forma individual. Después del inicio de la intensificación del tratamiento, la vigilancia para obtener información pronóstica adicional debe continuar. El aumento del monitoreo postoperatorio (monitoreo hemodinámico invasivo continuo) y el balance optimizado de oxígeno del miocardio (apoyo hemodinámico, corrección de la anemia y las alteraciones electrolíticas) pueden ser de beneficio adicional, pero actualmente sólo está respaldado por recomendaciones. Un estudio fármaco-económico mostró que la vigilancia de la Tnc debería ser rentable para todos los pacientes $\geq 45$ años que se sometieron a cirugía no cardíaca, pero sólo cuando se combina con una intervención activa una vez que se diagnostica el MINS ${ }^{(37)}$. En la Figura 4 se muestra el algoritmo para el manejo del MINS el cual es adaptado del propuesto por Biccard ${ }^{(38)}$ para el manejo del IM.

\section{CONCLUSIÓN}

El MINS ocurren con frecuencia en la CNC de los pacientes $>$ de 45 años de edad. El 10\% de los pacientes que presentan MINS fallecen en los 30 días posteriores a la cirugía. Se pueden diagnosticar fácilmente si se miden las Tnc en forma rutinaria en el postoperatorio como lo recomiendan las guías actuales internacionales. Una vez que se diagnostican, un simple tratamiento farmacológico puede mejorar los resultados; la valoración y el tratamiento de los MINS debe 
ser incorporado a la rutina del cuidado postoperatorio de los pacientes en riesgo de sufrir MINS.

El MINS es una condición adversa de gran magnitud y relevancia pronostica que afecta a ocho millones de pacientes en el mundo y se asocia con aproximadamente tres cuartos de millón de muertes anuales. Es probable que el número de pacientes afectados e identificados aumente tanto con el envejecimiento de la población como con la determinación de las Tnc de alta sensibilidad. Los mecanismos de MINS son poco conocidos, pero es probable que sean similares a los del IM tipo 1 y 2. Además, las medidas preventivas y los tratamientos aún no se han identificado de manera concluyente. Mientras tanto, la prevención de factores intraoperatorios y postoperatorios que pueden exacerbar la isquemia miocárdica y la reevaluación postoperatoria óptima de la terapia médica basada en las guías es una buena recomendación.

\section{REFERENCIAS}

1. Weiser TG, Haynes AB, Molina G, et al. Estimate of the global volume of surgery in 2012: an assessment supporting improved health outcomes. Lancet. 2015; 385: S11.

2. Group PS. Devereaux PJ, Yang H, et al. Effects of extended-release metoprolol succinate in patients undergoing non-cardiac surgery (POISE trial): a randomised controlled trial. Lancet. 2008; 371: 1839-1847.

3. Devereaux PJ, Mrkobrada M, Sessler DI, et al. Aspirin in patients undergoing noncardiac surgery. N Engl J Med. 2014;370:1494-1503.

4. Smilowitz NR, Berger JS. Perioperative management to reduce cardiovascular events. Circulation. 2016;133:1125-1130.

5. Devereaux PJ, Sessler DI. Cardiac complications in patients undergoing major noncardiac surgery. N Engl J Med. 2015;373:2258-2269.

6. Devereaux PJ, Chan MT, Alonso-Coello P, et al. Association between postoperative troponin levels and 30-day mortality among patients undergoing noncardiac surgery. JAMA. 2012;307:2295-2304.

7. Semel ME, Lipsitz SR, Funk LM, et al. Rates and patterns of death after surgery in the United States, 1996 and 2006. Surgery. 2012;151:171-182.

8. Ekeloef S, Alamili M, Devereaux PJ, et al. Troponin elevations after non-cardiac, non-vascular surgery are predictive of major adverse cardiac events and mortality: a systematic review and meta-analysis. Br J Anaesth. 2016;117:559-568.

9. Landesberg G, Shatz V, Akopnik I, et al. Association of cardiac troponin, CK-MB, and postoperative myocardial ischemia with long-term survival after major vascular surgery J Am Coll Cardiol. 2003;42:1547-1554

10. Kim LJ, MartínezEA, Faraday N, et al. Cardiac troponin I predicts short-term mortality in vascular surgery patients. Circulation. 2002;106:2366-2371.

11. Biccard BM. Detection and management of perioperative myocardial ischemia. Curr Opin Anaesthesiol. 2014;27:336-343.

12. Devereaux P. Characteristics and short-term prognosis of perioperative myocardial infarction in patients undergoing noncardiac surgery. Ann Intern Med. 2011;154:523-528.

13. Feigl EO. Coronary autoregulation. J Hypertens. 1989;7:S55-58.

14. Klocke FJ. Measurements of coronary flow reserve: defining pathophysiology versus making decisions about patient care. Circulation. 1987;76:1183-1189.

15. López HR, Poblano M, Sánchez C, Aguirre J, Franco J. Parámetros hemodinámicos y el tratamiento de choque como determinantes de la presión de perfusión coronaria (PPC) en el paciente crítico. Rev Asoc Mex Med Crit y Ter Int. 2010;24:119-123.

16. Cheatham M. Cálculos hemodinámicos. Disponible en www.surgicalcriticalcare.net/Lectures/hemodynamic_calculations.pdf2.

17. Barrera-Ramírez CF, Escaned J. Fisiología coronaria y su utilidad para el cardiólogo intervencionista: Medición invasiva de la presión y flujo coronarios. Arch Cardiol Mex 2005; 75:335-349.

18. Gobel FL, Norstrom LA, Nelson RR, et al. The rate-pressure product as an index of myocardial oxygen consumption during exercise in patients with angina pectoris. Circulation. 1978;57:549-556.

19. Sabate S, Mases N, Guilera J, et al. Incidence and predictors of major perioperative adverse cardiac and cerebrovascular events in non-cardiac surgery. Brith J Anesth. 2011;107:879-890.

20. Seller D, Srinivas C, Djaiani G. Cardiovascular complications after non-cardiac surgery. Anesthesia. 2018;73:34-42.
21. Smilowitz NR, Gupta N, Ramakrishna H, et al. Perioperative major adverse cardiovascular and cerebrovascular events associated with noncardiac surgery. JAMA Cardiol. 2017;2:181-187.

22. Levy M, Heels-Ansdell D, Hiralal R, et al. Prognostic value of troponin and creatine kinase muscle and brain isoenzyme measurement after noncardiac surgery: a systematic review and meta-analysis. Anesthesiology. 2011;114:796-806.

23. Redfern G, Rodseth RN, Biccard BM. Outcomes in vascular surgical patients with isolated postoperative troponin leak: a meta-analysis. Anaesthesia. 2011;66:604-610.

24. Thygesen K, Alpert JS, Jaffe AS, et al. Third universal definition of myocardial infarction. J Am Coll Cardiol. 2012;60:1581-1598.

25. Thygesen K, Alpert JS, Jaffe AS, et al. Four universal definition of myocardial infarction. Circulation. 2018;138:e618-e651.

26. Sandoval Y, Thordsen SE, Smith SW, et al. Cardiac troponin changes to distinguish type 1 and type 2 myocardial infarction and 180 day mortaliy risk. Eur Heart J Acute Cardiovasc Care. 2014;3:317-325.

27. Landesberg G, Beattie WS, Mosseri M, et al. Perioperative myocardial infarction. Circulation. 2009;119:2936-2944.

28. Gibbs NM, Crawford GP, Michalopoulos N. Postoperative changes in coagulant and anticoagulant factors following abdominal aortic surgery. J Cardiothorac Vasc Anesth. 1992;6:680-685.

29. Fukumoto Y, Hiro T, Fujii T, et al. Localized elevation of shear stress is related to coronary plaque rupture: a 3-dimensional intravascular ultrasound study with in-vivo color mapping of shear stress distribution. J Am Coll Cardiol. 2008;51:645-650.

30. Landesberg G. The pathophysiology of perioperative myocardial infarction: facts and perspectives. J Cardiothorac Vasc Anesth. 2003; 17:90-100.

31. Sandoval Y, Smith SW, Thordsen SE, et al. Supply/demand type 2 myocardial infarction: should we be paying more attention? J Am Coll Cardiol. 2014;63:2079-2087.

32. Lee TH, Marcantonio ER, Mangione CM, et al. Derivation and prospective validation of a simple index for prediction of cardiac risk of major noncardiac surgery. Circulation. 1999;100:1043-1049.

33. Gupta PK, Gupta H, Sundaram A, et al. Development and validation of a risk calculator for prediction of cardiac risk after surgery. Circulation2011;124:381-387.

34. Duceppe E. Canadian cardiovascular society guidelines on perioperative cardiac risk assessment and management for patients who undergo noncardiac surgery. Can J Cardiol. 2017;33:17-32.

35. Devereaux PJ, Duceppe E, Guyatt G, et al. Dabigatran in patients with myocardial injury after non-cardiac surgery (MANAGE): an international, randomised, placebo-controlled trial. Lancet. 2018;391:2325-2334.

36. Putzu A, de Carvalho E Silva CMPD, de Almeida JP, et al. Perioperative statin therapy in cardiac and non-cardiac surgery: a systematic review and meta-analysis of randomized controlled trials. Ann. Intensive Care. 2018;8:2-14.

37. Torborg A, Ryan L, Kantor G, et al. The pharmaco-economics of routine postoperative troponin surveillance to prevent and treat myocardial infarction after non-cardiac surgery. S Afr Med J. 2014;104:619-623.

38. Biccard BM. Perioperative myocardial infarction. S Afr J Anesth Analg. 2010;16:44-46. 